\section{Restoration of space-variant global blurs caused by severe camera movements and coordinate distortions}

To cite this article: Hakan Özaktas et al 1998 J. Opt. 29303

View the article online for updates and enhancements.
Related content
On the use of Cimmino's simultaneous projections method for computing a solution of the inverse problem in radiation therapy treatment planning
Y Censor, M D Altschuler and W D Powlis
- Inherent smoothness of intensity patterns for intensity modulated radiation therapy Ying Xiao, Darek Michalski, Yair Censor et al.
- Positive solutions to linear inverse problems
G D de Villiers, B McNally and E R Pike

\section{Recent citations}

- $\frac{\text { Parallel image restoration using surrogate }}{\text { constraint methods }}$
Bora Uçar et al
- Fast image recovery using dynamic load
$\frac{\text { balancing in parallel architectures, by }}{\text { means of incomplete projections }}$
J.L. Alba-Castro et al 


\title{
Restoration of space-variant global blurs caused by severe camera movements and coordinate distortions
}

\author{
Hakan Özaktaş, Mustafa Ç Pınar and Mustafa Akgül \\ Bilkent University, Faculty of Engineering, 06533 Bilkent, Ankara, Turkey \\ Received 8 April 1998, accepted 6 July 1998
}

\begin{abstract}
We show that a broad class of image recovery problems where an object undergoing an arbitrary two-dimensional, time- and space-variant, non-separable, nonlinear global coordinate distortion, is imaged for a certain duration, can be formulated as a system of linear inequalities. Since the system of inequalities arising in this context can be solved efficiently, our approach yields an effective method for solving this class of image recovery problems. A novel step size policy is introduced to accelerate the parallel surrogate constraint algorithm employed. The approach is illustrated by recovering an image severely blurred by the combined effects of translational and rotational motion and elliptic scaling.
\end{abstract}

Keywords: Image recovery, image restoration, deblurring, linear feasibility, surrogate constraint algorithms

\section{Restauration des distortions globales à cause des mouvements sévères de coordonnées}

Résumé. Nous démontrons qu'il est possible de reformuler un vaste groupe de problèmes de récupération d'image comme un système d'inégalités linéaires de grande dimension. Il s'agit en général, d'une image dans laquelle un objet subit pendant une certaine durée une distortion arbitraire, globale, nonséparable, nonlinéaire de coordonnées en deux dimensions. Nous résolvons le systéme d'inégalités au moyen d'un nouvel algorithme de multiprojections simultanées. L'application de la méthodologie est illustrée sur l'exemple d'une image sujet à une distortion sévère sous un mouvement de trajet compliqué.

Mots clés: Récupération d'image, restauration d'image, systèmes d'inégalités linéaires, algorithmes de multiprojections

\section{Introduction}

In this study, we show that a rather general class of image recovery problems can be solved efficiently by formulating them as a linear feasibility problem (system of linear inequalities). These problems arise when an original image or object undergoing an arbitrary two-dimensional, timeand space-varying, non-separable and nonlinear global coordinate distortion is imaged by a camera or other device and it is desired to recover the original image. We also present a new parallel surrogate constraint algorithm which employs a new step size policy to solve the resulting feasibility problem.

When discretized, the class of problems which we deal with is of the general linear form:

$$
g=H f
$$

where $g$ is the vector representing the observed image and $f$ is the vector representing the original image to be recovered. $H$ represents the system which distorts the image. If the images have $N \times N$ pixels, then $f$ and $g$ are column vectors of size $N^{2}$ obtained by stacking the columns of the image matrix on top of each other [1]. Thus $H$ is an $N^{2} \times N^{2}$ matrix. (Generalization to rectangular images is straightforward.) For the $160 \times 160$ images used in our examples, the matrix $H$ has a size of $25600 \times 25600$. Normally, matrices of this and larger sizes cannot be handled unless they are sparse. The class of problems we deal with lead to such large, sparse matrices representing distortions and blurs which are:

(i) Nonseparable. The two dimensions are coupled and the problem cannot be reduced to two one-dimensional problems. 
(ii) Anisotropic. The distortion is different along different directions.

(iii) Space variant. The distortion is different for different parts of the image; it is not space invariant.

(iv) Nonlocal. The value of the distorted image at a certain point may depend on values of the original image at distant points; the distortion is of a global nature.

If the system was separable or isotropic or space invariant, it would have been possible to simplify the problem by exploiting the special structure of $H$. (For instance, see image processing texts such as [1-3]. Also see [4] for a discussion of the difficulties involved in the general case and an approach appropriate when the distortion is local.) In our case, the matrix $H$ does not exhibit any special structure which would allow some reduction or decomposition techniques to be used. Furthermore, due to the large size of $H$, direct methods such as Wiener filtering or those based on singular-value decomposition would require too much storage and time. Despite these difficulties, the approach taken in this paper allows such problems to be solved efficiently.

In the next section we formulate the class of distortion and blur problems addressed in this paper and show how they can be posed as a feasibility problem. The negative effects of noise and how to reduce them are also discussed. As an example, we consider the restoration of an image blurred by the combined effects of translational and rotational motion, and elliptic scaling. Then we briefly discuss the sequential and parallel surrogate constraint algorithms for solving the feasibility problem. We also introduce a novel step size policy for speeding up the implementation of the parallel surrogate constraint algorithm.

\section{Formulation of the problem}

Consider an original image $f(q)$ which undergoes a general time-varying, nonlinear coordinate distortion represented by $q=\rho(r, t)=[u(r, t), v(r, t)]$ so that at time $t$ the image is observed as $f(\rho(r, t))=f(u(r, t), v(r, t))$. Here $r$ stands for the position vector $(x, y)$. Now assume that we expose a photographic film to this time-varying image for a duration of $T$ seconds. The image $g(r)$ recorded on the film is given by

$$
g(r)=K \int_{t=0}^{T} f(\rho(r, t)) \mathrm{d} t
$$

where $K$ is a constant. (This equation may also have other physical interpretations than the one mentioned here. One such interpretation-the image reconstruction from projections problem-will be mentioned later.) To appreciate the generality of this observation model, we consider some of its special cases:

(i) Translational motion. $\rho(r, t)=r-r_{c}(t)$ where $r_{c}(t)=\left[x_{c}(t), y_{c}(t)\right]$ is a given function representing the motion of the original image or object as a function of time. Arbitrary two-dimensional movement with arbitrary acceleration and higher-order derivatives is allowed. (Although we speak of the movement of the object, it is clear that this also covers movement of the film or camera.) (ii) Isotropic scaling. $\rho(r, t)=r / m(t)$ where $m(t)$ is an arbitrary scaling function of time. More generally, different scaling functions can be assumed in the two dimensions (anisotropic or elliptic scaling). By properly choosing and interpreting $m(t)$, it is possible to model the movement of the object towards or away from the camera.

(iii) Rotation. $\rho(r, t)=R_{\phi(t)} r$ where $R_{\phi(t)}$ is the $2 \times 2$ rotation matrix $[\cos \phi(t), \sin \phi(t) ;-\sin \phi(t), \cos \phi(t)]$. Here $\phi(t)$ is an arbitrary function of time representing the angle of rotation. More generally, the $2 \times 2$ matrix may take the form of an arbitrary non-orthogonal (parallelogram-type) distortion represented by the matrix $[a(t), b(t) ; c(t), d(t)]$, which can be used to model moderate out of plane rotations of the object.

Other special cases and their combinations may also be considered.

Since equation (2) represents a linear relation between $g$ and $f$, it is possible to write it in the form

$$
g(r)=\int_{r^{\prime}} H\left(r, r^{\prime}\right) f\left(r^{\prime}\right) \mathrm{d} r^{\prime} .
$$

To find $H\left(r, r^{\prime}\right)$, we use

$$
f(\rho(r, t))=\int_{r^{\prime}} f\left(r^{\prime}\right) \delta\left(r^{\prime}-\rho(r, t)\right) \mathrm{d} r^{\prime}
$$

in equation (2) to obtain

$$
g(r)=\int_{r^{\prime}}\left[K \int_{t=0}^{T} \delta\left(r^{\prime}-\rho(r, t)\right) \mathrm{d} t\right] f\left(r^{\prime}\right) \mathrm{d} r^{\prime}
$$

from which we conclude that

$$
H\left(r, r^{\prime}\right)=K \int_{t=0}^{T} \delta\left(r^{\prime}-\rho(r, t)\right) \mathrm{d} t .
$$

The discrete counterpart of equation (3) is simply the general linear matrix equation $g=H f$. Since equation (5) is a one-dimensional integral over time, whereas the images are two dimensional, the number of non-zero elements of $H$ will typically be of the order of $N^{3}$ (an average of $N$ elements per row) out of $N^{2} \times N^{2}=N^{4}$ elements. This sparsity property is what makes it possible to solve this class of problems, despite the fact that the dimensions of $H$ are very large. The large and sparse matrix $H$ is represented in sparse matrix format [5]. Since $\rho(r, t)$ is arbitrary, the non-zero elements of $H$ will, in general, be distributed quite irregularly and will not exhibit any special structure. We emphasize that unlike many sparse matrix algorithms which rely on some special structure of the matrix, the approach we employ is particularly suited and effective in handling matrices whose non-zeros are arbitrarily distributed.

Typically there will be an amount of measurement error or noise associated with the observation, leading to an inconsistent system of equations. Denoting the noisy observation by $g^{\prime}$, we allow for such errors by writing

$$
\left|\left(g^{\prime}-H f\right)_{i}\right| \leq \epsilon \quad i=1, \ldots, N^{2}
$$

where $\left(g^{\prime}-H f\right)_{i}$ is the $i$ th component of $g^{\prime}-H f$ and $\epsilon$ is a suitable error tolerance parameter, which we will quote as a percentage of the mean value of $g$. 
Equation (6) can be converted into a feasibility problem of the form $A \xi \leq b$ by setting

$$
\begin{aligned}
& A=\left[\frac{H}{-H}\right]_{2 N^{2} \times N^{2}} \\
& b=\left[\frac{\mathcal{E}+g^{\prime}}{\mathcal{E}-g^{\prime}}\right]_{2 N^{2} \times 1} \\
& \xi=f_{N^{2} \times 1}
\end{aligned}
$$

where $\mathcal{E}$ is an $N^{2} \times 1$ vector of $\epsilon$ 's.

Although the solution of a linear inequality system of the form $A \xi \leq b$ with conventional (Kaczmarzor Cimmino-type) projection algorithms is in principle straightforward, convergence is often exceedingly slow for large problems. This has led to the development of the block projections or surrogate constraint algorithms [6]. The sequential surrogate constraint algorithm performs much better than the conventional projection-onto-convexsets (POCS) algorithms [7]. In contrast, the parallel surrogate constraint algorithm has been found to be inferior [8], so that it has not been possible to benefit from parallel processing. However, we have shown that adjustment of the step size policy in the parallel algorithm leads to accelerated convergence [9], making this an attractive option. Discussion of these algorithms for finding a vector $\xi$ which satisfies $A \xi \leq b$ is postponed to section 5 .

\section{Pre-filtering and smoothing}

When the relative measurement errors are very small, the solution of equation (6) may directly yield a good restoration of the original image $f$. However, in general, since we are dealing with a severely ill-conditioned problem [10], even small observation errors will tend to show up as very large restoration errors. It is thus necessary to introduce some kind of regularization [10] to limit the negative effects of noise to what is fundamentally unavoidable. Physically, what happens is that the severe distortions we consider blur the finer details of the original image and those image components which are depressed below the noise level become irretrievably lost. However, unless care is exercised the restoration process may amplify this noise, resulting in a restored image which is much worse than the best possible.

There are many approaches to regularizing illconditioned inverse problems, most of which involve the introduction of some kind of a priori knowledge regarding the original image. This knowledge may take the form of additional constraints or statistical information. We will assume that such information is not available apart from the condition of piecewise smoothness, which means that the image consists of smooth regions separated by sharp edges. This is the most we will allow ourselves since our interest is in generic real images, for which even this condition is only partly valid.

Our regularization procedure consists of two components. In the first, called pre-filtering, we try to reduce the noise in $g^{\prime}$ as much as possible. We multiply the Fourier transform of $g^{\prime}$ by $\left(1+S_{n} / S_{g}\right)^{-1}$, where $S_{n}$ is the power spectral density of the noise (simply equal to the variance for zero mean white Gaussian noise) and $S_{g}$ is the magnitude squared of the Fourier transform of $g$. When $S_{n} \ll S_{g}$, the noise is small so that the filter is simply equal to 1 . When $S_{n} \gg S_{g}$, noise is dominant and the filter is close to 0 . In between, the filter effects a smooth transition. Thus, the overall effect of this filter is to eliminate the components of the image for which noise is dominant, while preserving others. Of course, in reality we do not have access to $g$, but only $g^{\prime}$. Thus we have used $S_{g^{\prime}}-S_{n}$ as a surrogate for $S_{g}$ (setting it equal to zero for those frequencies where it goes negative). This is based on the assumption that the noise is not correlated with $g$ so that $S_{g^{\prime}}=S_{g}+S_{n}$.

The second component of our regularization procedure, smoothing, is applied after the feasibility problem is solved and a tentative (noisy) restoration $f_{\text {tent }}$ is obtained. We introduce the following edge-preserving nearest neighbour smoothing, inspired by edge-preserving penalty functions [11]:

$$
f_{\text {tent }} \rightarrow f_{\text {tent }}+\beta \Delta f_{\text {tent }} z\left(\Delta f_{\text {tent }} / \gamma\right)
$$

where $0<\beta \leq 0.5, \gamma>0, z(\zeta)=\arctan (\zeta) / \zeta$ and $\Delta f_{\text {tent }}$ is the difference between the average of the four nearest neighbours of a given image pixel and the pixel itself. The usual choice for $\beta$ is 0.5 and the usual choice for $\gamma$ is comparable or somewhat larger than the level of noise which we are trying to smooth out, but sufficiently smaller than the edges or other features we are trying to protect. (Naturally, these two requirements sometimes conflict for the generic images we are considering, so that a compromise is necessary.) When $\Delta f_{\text {tent }} / \gamma \ll 1$, $z\left(\Delta f_{\text {tent }} / \gamma\right) \approx 1$ and the procedure becomes equivalent to taking the average of a given image pixel with the average of its neighbours. When $\Delta f_{\text {tent }} / \gamma \gg 1, z(\Delta f / \gamma)$ is small and the procedure results in little change. A third parameter of the smoothing process is the number of times $(n)$ equation (8) is applied consecutively. The overall effect is to smooth out the noise, while limiting the damage to the edges.

\section{Example}

As an example, we will consider restoration of an image distorted by the combined effects of translational and rotational motion and elliptic scaling:

$$
\begin{aligned}
& u(x, y, t)=\frac{\cos \phi(t)\left(x-x_{c}(t)\right)+\sin \phi(t)\left(y-y_{c}(t)\right)}{m_{u}(t)} \\
& v(x, y, t)=\frac{-\sin \phi(t)\left(x-x_{c}(t)\right)+\cos \phi(t)\left(y-y_{c}(t)\right)}{m_{v}(t)}
\end{aligned}
$$

where $u(x, y, t)$ and $v(x, y, t)$ are components of $\rho(r, t)$. We emphasize that there is nothing special about this particular example; the present approach can equally efficiently handle arbitrary nonlinear $\rho(r, t)$, which may even be discontinuous and fragmented. We will consider two cases. The functions $x_{c}(t)$ and $y_{c}(t)$ which define the translational motion are given in figure 1 along with the trajectory itself. Notice that the motion can be quite 

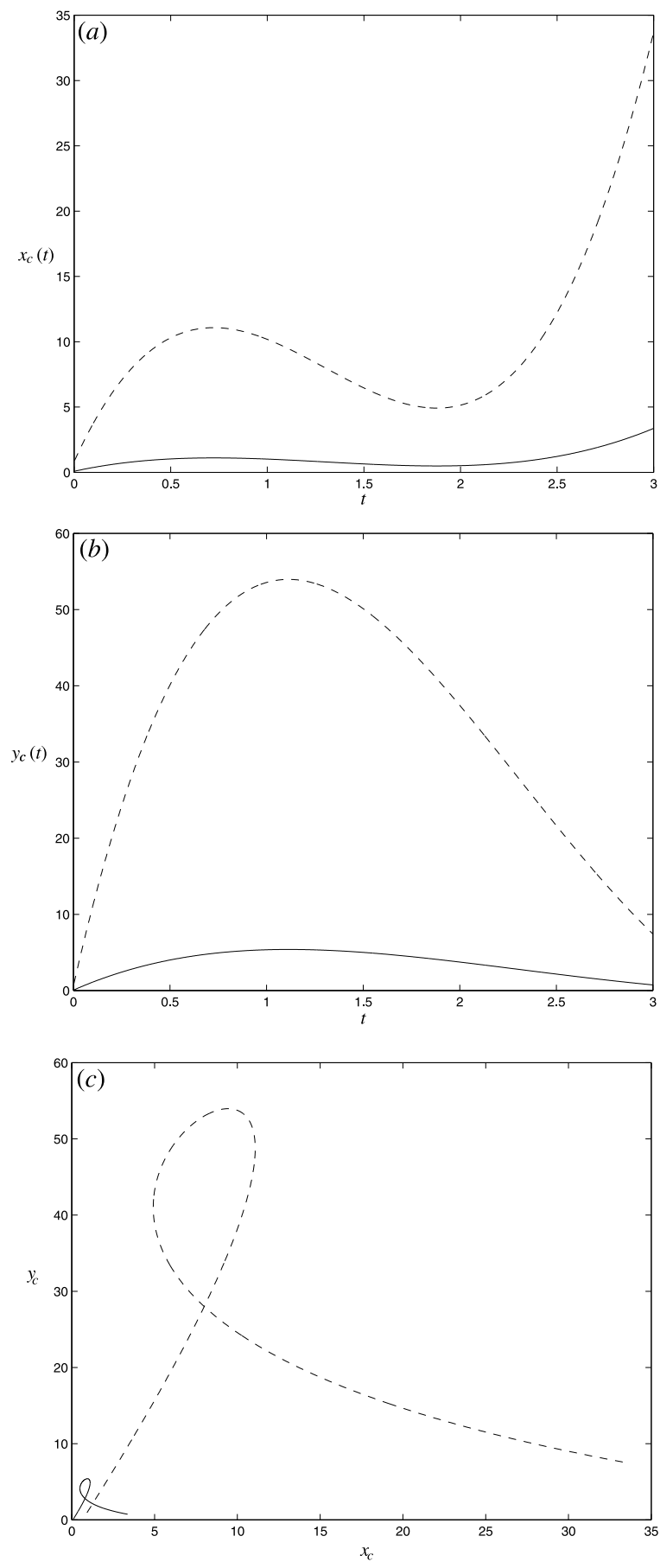

Figure 1. (a) $x_{c}(t) ;(b) y_{c}(t) ;(c)$ the complete trajectory from $t=0$ to $t=T=3$. $x$ and $y$ are measured in a number of image pixels (case (i), full curve; case (ii), broken curve).

irregular and involves not only acceleration but also higherorder derivatives. In case (i), the extent of the motion is small in comparison to the size of the images, whereas in case (ii) it is comparable to the size of the images. The functions $\phi(t), m_{u}(t)$ and $m_{v}(t)$ are illustrated in figure 2 . In case (i), the object undergoes a moderate rotation of $5^{\circ}$, whereas in case (ii) it undergoes a $180^{\circ}$ rotation and ends upside down. Thus while case (i) represents a moderate local blur, in case (ii) the image undergoes a severe blurring
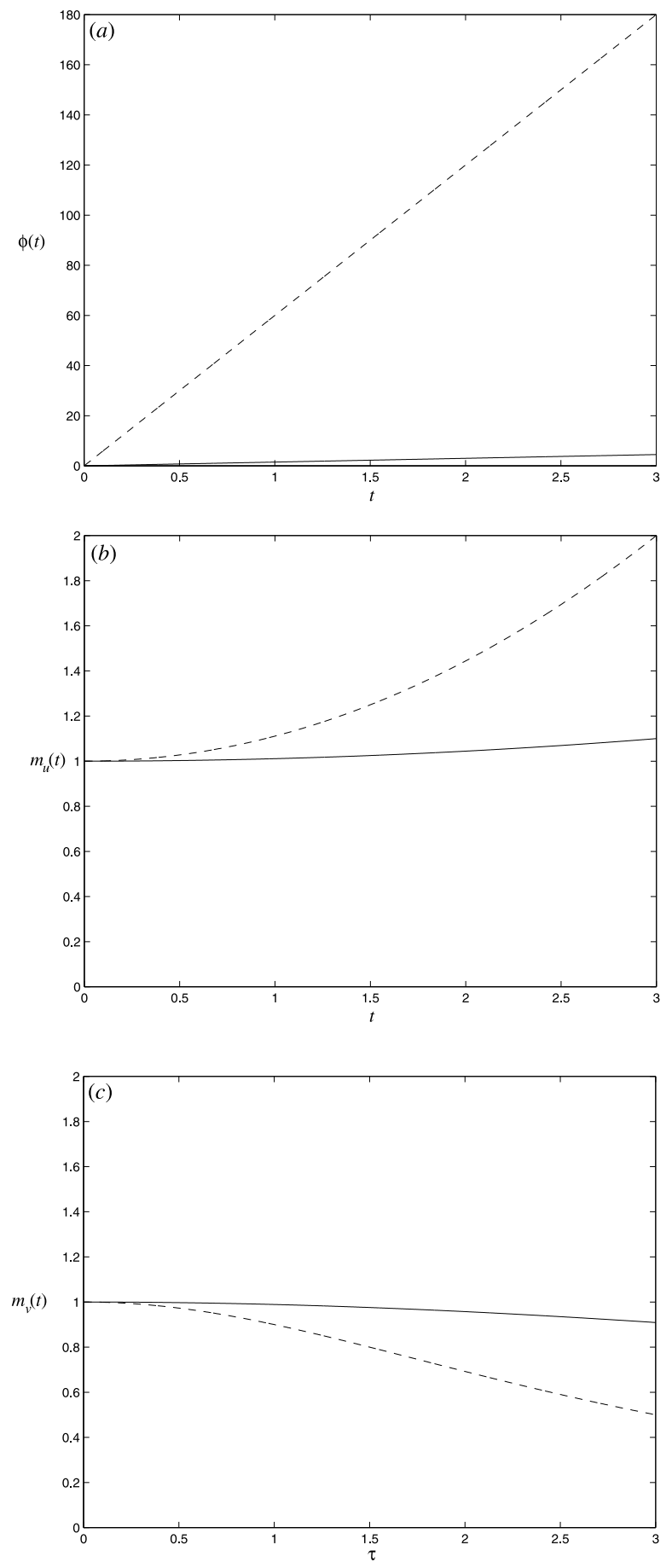

Figure 2. (a) $\phi(t) ;(b) m_{u}(t) ;(c) m_{v}(t)$ (case (i), full curve; case (ii), broken curve).

of a global nature. The original and the distorted images are shown in figure 3 . These were obtained by discretizing the time interval $T$ in equation (2) into 2048 points and using linear interpolation. The measurement errors were simulated by adding identically independent distributed zero-mean normal random variables to $g$ such that $\epsilon=$ 2 standard deviations. After pre-filtering the observation, the recovery problem can be formulated as a feasibility problem of the form $A \xi \leq b$ as discussed in section 2 and 

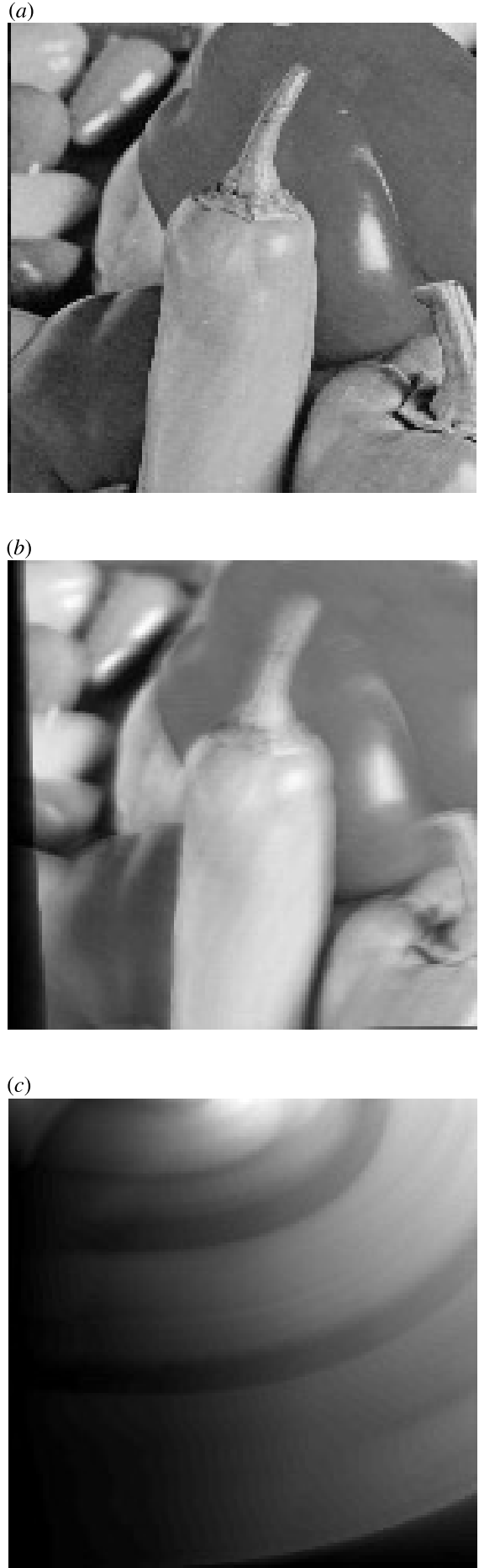

Figure 3. (a) Original image; (b) distorted image (case (i)); (c) distorted image (case (ii)).

the feasibility problem can be solved by using the class of algorithms discussed in the next section. Finally, smoothing is employed to obtain the recovered images shown in figure 4 . Table 1 shows the resulting mean restoration errors as a percentage of the mean image intensity.

In case (i), the distortion is moderate so that good restorations are possible even when the measurement error is relatively large. Although the distortion in case (ii)
Table 1. Restoration errors for case (i) (moderate blur) and case (ii) (severe blur) for two different levels of the measurement error, quoted as a percentage of the mean value of $g$. The numbers in the parentheses are the values of $n, \beta$ and $\gamma$ used, where $\gamma$ is expressed as a percentage of the mean image intensity.

\begin{tabular}{lll}
\hline & $0.5 \%$ & $5 \%$ \\
\hline case (i) & $1.9 \%(1,0.5,5 \%)$ & $5.3 \%(2,0.5,10 \%)$ \\
case (ii) & $5.6 \%(4,0.5,10 \%)$ & $12.9 \%(12,0.5,30 \%)$ \\
\hline
\end{tabular}

is very severe, blurring the original beyond any possible recognition, quite good restorations are obtained when the noise is small. However, the restorations become less satisfactory as the measurement error is increased. This illustrates the fundamental trade-off between the amount of noise and blur which can be tolerated simultaneously in illconditioned problems. When the noise and blur are both large, a significant amount of information becomes lost and a faithful restoration is not possible.

Further improvements would be possible by introducing additional a priori knowledge in the form of additional constraints or statistical information, which we have assumed are not available. The condition of piecewise smoothness we have employed is only approximately valid for the generic images we have considered. This condition is much more effective when the original images consist of relatively large regions separated by sharp boundaries and when the intensity within each region is constant or nearly constant [11].

\section{Algorithms for solving the feasibility problem}

In this section we briefly review surrogate constraint algorithms for solving $A \xi \leq b$. These algorithms can effectively handle situations where $A$ is a very large sparse matrix without any special pattern and whose non-zero elements are distributed irregularly. For this reason, these algorithms are particularly suited to the class of problems under consideration. This match between problem and solution is the main rationale behind the approach taken in this paper. Readers without a particular interest in these algorithms may skip this section.

The theoretical foundations of the projections approach are established in the historical works $[12,13]$. The iterative algorithm, which considers the orthogonal projection onto violated inequalities one at a time, is referred to as the relaxation (also known as POCS: projection-onto-convexsets) method. In later years, a method known as the surrogate constraint algorithm (also referred to as the block projections approach) has been developed $[6,7,14]$. In this approach, one considers the projection of the iteration point onto many violated constraints at a time, instead of onto a single unsatisfied inequality.

For typical image processing problems (image sizes of the order of $10^{5}$ pixels) surrogation becomes a necessity since the relaxation (or POCS) method is far from being practical. It is reported that even for smaller problems, the surrogate constraint algorithms outperform the relaxation method [7]. 

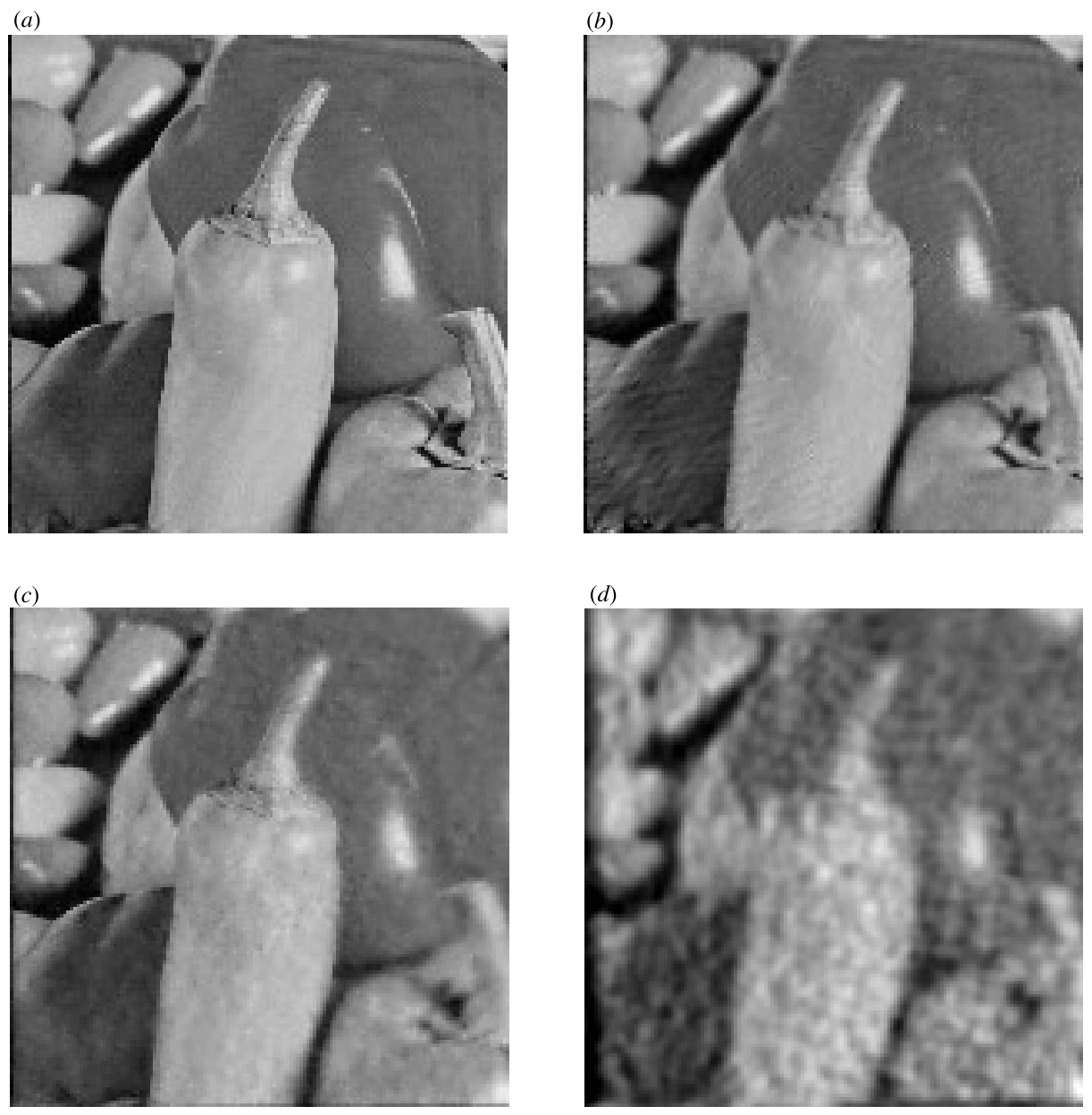

Figure 4. Recovered images: (a) case (i) $(0.5 \%)$; (b)

b) case (i) (5\%); (c) case (ii) $(0.5 \%)$; (d) case (ii) $(5 \%)$.

The projection of a point $\xi$, onto a convex set $S$, is the point in $S$ which is closest to $\xi$. (In this paper convex sets are defined by sets of linear inequalities.) Projection algorithms may be broadly classified as being sequential or parallel. In sequential algorithms the iterate is projected successively onto the constraints. In parallel algorithms a weighted combination of the projections is employed instead. As their name implies, parallel algorithms are particularly suited for implementation on parallel computers.

We now briefly outline the surrogate constraint (or block projections) approach. Consider the partitioning of the matrix $A$ and the column vector $b$ into $p$ row-wise blocks of equal or almost equal sizes, as $\left[A_{1}\left|A_{2}\right| \ldots \mid A_{p}\right]^{\mathrm{T}}$ and $\left[b_{1}\left|b_{2}\right| \ldots \mid b_{p}\right]^{\mathrm{T}}$. (In the case of parallel implementation $p$ is equal to the number of processors.) The iteration begins by setting the initial value $\xi^{0}$ equal to a column of zeros. Below, we describe how to obtain the next iterate $\xi^{k+1}$ from $\xi^{k}$, where $k$ denotes the iteration index.

The projection of $\xi^{k}$ onto the $\kappa$ th block of constraints $A_{\kappa} \xi \leq b_{\kappa}$, denoted as $P_{\kappa}\left(\xi^{k}\right)$, can be computed with the following formula [7]:

$$
P_{\kappa}\left(\xi^{k}\right)=\xi^{k}-d_{\kappa}^{k}
$$

where

$$
d_{\kappa}^{k}=\frac{\left(\pi_{\kappa}^{k} A_{\kappa} \xi^{k}-\pi_{\kappa}^{k} b_{\kappa}\right)\left(\pi_{\kappa}^{k} A_{\kappa}\right)}{\left\|\pi_{\kappa}^{k} A_{\kappa}\right\|^{2}}
$$

where $\pi_{\kappa}^{k}$ is a row vector whose $i$ th component is equal to 1 if the $i$ th constraint is violated and is equal to 0 otherwise. (The $i$ th constraint is simply the $i$ th row of the matrix inequality $A \xi \leq b$.) In the event that a particular block of constraints is fully satisfied we take $d_{\kappa}^{k}=0$ so that $P_{\kappa}\left(\xi^{k}\right)=\xi^{k}$.

Now we form the following weighted projection:

$$
P\left(\xi^{k}\right)=\sum_{\kappa=1}^{p} \tau_{\kappa} P_{\kappa}\left(\xi^{k}\right)=\sum_{\kappa=1}^{p} \tau_{\kappa}\left(\xi^{k}-d_{\kappa}^{k}\right)
$$

where $\tau_{\kappa}$ are weight coefficients satisfying $\sum_{\kappa=1}^{p} \tau_{\kappa}=1$ and $\tau_{\kappa} \geq 0$. In addition, one should take $\tau_{\kappa}>0$ for any violated block to ensure convergence [7].

Finally, the next iteration point is generated as

$$
\xi^{k+1}=\xi^{k}+\lambda_{k}\left(P\left(\xi^{k}\right)-\xi^{k}\right)
$$

where $0<\lambda_{k}<2$. Using equation (12) this reduces to

$$
\xi^{k+1}=\xi^{k}-\lambda_{k}\left(\sum_{\kappa=1}^{p} \tau_{\kappa} d_{\kappa}^{k}\right) .
$$


When $\lambda_{k}=1$ we obtain $\xi^{k+1}=P\left(\xi^{k}\right)$ : the next iteration point is simply the weighted projection. However in general, choice of other values of $\lambda_{k}$ is found to speed up the algorithm. This algorithm generates a sequence $\xi^{k}$, which converges to a point in the solution set as $k \rightarrow \infty$ (provided that it is non-empty). In practice, the algorithm is terminated when all of the constraints are satisfied within a specified tolerance [7].

The given algorithm is said to be one of cyclic control (corresponding to the sequential surrogate constraint algorithm given in [7]) if at each iteration only one block coefficient is non-zero $\left(\tau_{\kappa}=1\right.$ for a single $\left.\kappa\right)$ and at the following iteration only the block coefficient with the successive index is non-zero $\left(\tau_{\kappa+1}=1\right)$. If this is the case, the unnecessary computations related to blocks with zero coefficients are not made. For the parallel surrogate constraint algorithm of Yang and Murty, in the absence of additional information, it is natural to take equal weights $\tau_{\kappa}$ for all blocks whose inequalities are not fully satisfied (since none of the blocks has priority with respect to the others) and zero weights for the blocks whose inequalities are fully satisfied.

Unfortunately, the parallel Yang-Murty algorithm performs much worse than its sequential counterpart, even when implemented on parallel processors. For this reason we have recently suggested the following modification of the step size policy which yields much better results [9]. Instead of taking $\xi^{k+1}$ according to equation (14), we have produced the next iterate according to

$$
\xi^{k+1}=\xi^{k}-\lambda_{k}\left(\frac{\sum_{\kappa=1}^{p}\left\|d_{\kappa}^{k}\right\|^{2}}{\left\|\sum_{\kappa=1}^{p} d_{\kappa}^{k}\right\|^{2}} \sum_{\kappa=1}^{p} d_{\kappa}^{k}\right)
$$

The reader is referred to [9] for a detailed explanation of this strategy as well as performance results on a Parsytec CC-24 parallel computer.

\section{Discussion and conclusion}

In this paper we have shown that a rather general class of image blurring and distortion problems can be formulated as a linear feasibility problem of the form $A \xi \leq b$. The feasibility problem approach has been used previously for medical image reconstruction (computerized tomography) [15]. It is not difficult to see that the tomography problem [1] is a special case of our formulation (see equation (2)):

$$
g(s, \theta)=\int_{t} f(\rho(s, \theta, t)) \mathrm{d} t=\int_{t} f(u(s, \theta, t), v(s, \theta, t)) \mathrm{d} t
$$

where

$$
\begin{aligned}
& u(s, \theta, t)=s \cos \theta-t \sin \theta \\
& v(s, \theta, t)=s \sin \theta+t \cos \theta .
\end{aligned}
$$

Here $t$ is no longer time but the integration variable along the path of x-rays. We emphasize that in this paper we allowed $u(r, t)$ and $v(r, t)$ to be arbitrary nonlinear functions, which may even be discontinuous and fragmented.

The class of problems we have addressed are in general non-separable, anisotropic, global and space variant.
Furthermore, it is not assumed that the space variance is of a slowly varying nature; so that the present approach is able to handle cases of extreme or abrupt space variance as well. (Many treatments of space-variant problems make a slowly varying assumption so that the problem can be approximately reduced to many smaller space-invariant problems.)

The arbitrary and global nature of $\rho(r, t)$ results in large, sparse matrices (with the order of $10^{9}$ elements) whose non-zeros are irregularly distributed. The surrogate constraint algorithms employed are particularly suited for such situations. This match between a difficult problem and its effective solution is the main motivation of the approach taken in this paper.

There exists a fundamental trade-off between the amounts of distortion and noise that can be tolerated simultaneously in an ill-conditioned problem. The approach presented can be especially useful on that side of this tradeoff where the distortion is of a severe and very irregular nature, but the noise is low or moderate.

A number of generalizations of this approach can be readily implemented. If the imaging device has an arbitrary space-varying point spread function (PSF) of limited extent, then its effects can also be incorporated into the matrix $H$. The only limitation arises from the fact that this will increase the number of non-zero elements of $H$ (especially if the PSF is relatively broad), resulting in memory problems. What ultimately matters is the largest number of non-zero elements that the computer can handle. Another advantage of this approach is that it is possible to accommodate situations in which the observed data are incomplete or partial, or when additional a priori information about the original image exists.

\section{Acknowledgments}

The authors would like to thank Alper Kutay and Haldun M Özaktaş for many useful discussions and suggestions, which were indispensable in formulating and clarifying the ideas presented in this paper.

\section{References}

[1] Jain A K 1989 Fundamentals of Digital Image Processing (Englewood Cliffs, NJ: Prentice-Hall)

[2] Lim J S 1990 Two-Dimensional Signal and Image Processing (Englewood Cliffs, NJ: Prentice-Hall)

[3] Pratt W K 1991 Digital Image Processing 2nd edn (New York: Wiley)

[4] Fish D A, Grochmalicki J and Pike E R 1996 Scanning singular-value decomposition method for restoration of images with space-variant blur J. Opt. Soc. Am. 13 464-9

[5] Pissanetzky S 1984 Sparse Matrix Technology (London: Academic)

[6] Aharoni R and Censor Y 1989 Block-iterative projection methods for parallel computation of solutions to convex feasibility problems Linear Algebra Applic. 120 165-75

[7] Yang K and Murty K G 1992 New iterative methods for linear inequalities J. Optimiz. Theory Applic. 72 163-85 
[8] Özaktaş H, Akgül M and Pınar M Ç 1996 The parallel surrogate constraint approach to the linear feasibility problem Lecture Notes in Computer Science 1184 ed J Waśniewski, J Dongarra, K Madsen and D Olesen (Berlin: Springer) pp 565-74

[9] Özaktaş H, Pınar M Ç and Akgül M 1997 An improved simultaneous block projections algorithm for the linear feasibility problem Proc. 12th Int. Symp. Computer and Information Sciences ed S Kuru, M U Çağlayan and H L Akın (Istanbul: Boğaziçi University) pp 551-8

[10] Rushforth C K 1987 Signal restoration, functional analysis and Fredholm integral equations of the first kind Image Recovery: Theory and Application ed H Stark (Orlando, FL: Academic) pp 1-27
[11] Delaney A H and Bresler Y 1998 Globally convergent edge-preserving regularized reconstruction: an application to limited-angle tomography IEEE Trans. Image Process. 7 204-21

[12] Agmon S 1954 The relaxation method for linear inequalities Can. J. Math. 6 382-92

[13] Motzkin I S and Schoenberg I J 1954 The relaxation method for linear inequalities Can. J. Math. 6 393-404

[14] Kiwiel K C 1995 Block-iterative surrogate projection methods for convex feasibility problems Linear Algebra Applic. 215 225-60

[15] Herman G T 1980 Image Reconstruction from Projections (New York: Academic) 